\title{
DIVERSIDAD MORFOLÓGICA DEL FRUTO EN GERMOPLASMA NATIVO DE MANGO Mangifera indica L. (ANACARDIACEAE)
NATIVO DE CHIAPAS, MEXICO
}

\section{Gálvez-López ${ }^{1}$, M. Salvador-Figueroa ${ }^{2} y$ N. Mayek-Pérez ${ }^{1^{*}}$}

I Centro de Biotecnología Genómica, Instituto Politécnico Nacional. Blvd. del Maestro esquina Elías Piña s/n, Col. Narciso Mendoza, Reynosa, Tamaulipas, 887 io. México.

2 Área de Biotecnología, Universidad Autónoma de Chiapas. Carretera a Puerto Madero KM. 2.o. Tapachula, Chiapas, 30700. MÉXico.

* E-MAIL: NMAYEK@IPN.MX

\section{RESUMEN}

El cultivo del mango es parte importante del desarrollo económico del estado de Chiapas, México donde este frutal se cultiva en aproximadamente 28.000 ha. La recombinación entre los diferentes germoplasmas introducidos en la región ha resultado en una amplia gama de mangos, cuyas características agronómicas podrían aprovecharse para el mejoramiento genético. En este trabajo se estudió la diversidad morfológica del fruto de 36 mangos nativos de Chiapas. Veintiocho características morfológicas de fruto y semilla de cada accesión se midieron de acuerdo con descriptores internacionales y a dicha información se calcu- laron parámetros estadísticos. Se observó amplia diversidad en características morfológicas del fruto, particularmente en color de cáscara (ocho clases), peso del fruto (127 a $498 \mathrm{~g}_{\text {fruto }^{-1}}$ ) y grosor del mesocarpio $(0,9$ a $5,2 \mathrm{~cm})$. Los mangos "Ajo" y "Sin nombre-2" de la localidad de Tuxtla Chico mostraron características del fruto similares a las de la variedad "Ataulfo" (la variedad más cultivada en Chiapas por su calidad). Dado que los mangos nativos son fuente de riqueza genética de características deseables para el cultivo comercial y para el mejoramiento genético, es necesario establecer trabajos para su conservación y propagación.

Palabras clave: Mangifera indica L., región Soconusco, recursos fitogenéticos. 


\section{INTRODUCCIÓN}

El estado de Chiapas, México es rico en biodiversidad de mangos que se ha incrementado con el clima del trópico húmedo que ha estimulado el desarrollo de especies endémicas de plantas y animales con características únicas como es el caso del mango (Mangifera indica L.). En la región del Soconusco, ubicada en el sureste de Chiapas, existe una amplia gama de tipos de mangos nativos cuya denominación es local y cuyo valor comercial y/o ecológico aún no se ha cuantificado. Dichos mangos podrían ser portadores potenciales de genes que pudieran aprovecharse en el mejoramiento genético de la especie a fin de resolver problemáticas locales tales como el tamaño del árbol, la producción irregular de frutos, el amarre escaso de frutos y la sensibilidad al frío, plagas y enfermedades (CHÁVEZ, 2001). Por lo anteriormente expuesto, es necesario iniciar con estudios que permitan conocer el potencial genético de cada tipo de mango nativo para establecer las estrategias de mejoramiento más apropiadas. La caracterización de una colección de germoplasma consiste en registrar y describir sistemáticamente las accesiones de la misma especie con base en aquellas características cualitativas y cuantitativas altamente heredables, las que se observan fácilmente y se expresan en la mayoría de los ambientes de prueba (JARAMILLO \& BAENA, 2000). La descripción morfológica permite conocer al germoplasma y determinar su utilidad potencial. Los descriptores deben ser específicos para cada especie, capaces de diferenciar entre genotipos y expresar el atributo de manera precisa y uniforme. Varios atributos pueden describir un material genético, pero los caracteres realmente útiles son aquellos que pueden detectarse a simple vista y registrarse fácilmente; mostrar alta heredabilidad y alto valor taxonómico y agronómico, que se pueden aplicar a muestras pequeñas y que permitan diferenciar una accesión de otra (IPGRI, 1989). El Instituto Internacional de Recursos Genéticos Vegetales (International Plant Genetic Resources Institute, IPGRI; Roma, Italia) ha establecido una lista de descriptores para mango que incluye características morfológicas de planta, hojas, flores y frutos. Esta lista constituye un formato universal para la caracterización de los recursos genéticos de la especie con la finalidad de obtener datos concretos y confiables sobre la identificación y caracterización de una variedad o genotipo de interés (IPGRI, 1989). En el mundo se han caracterizado morfológicamente diversas variedades de mango de interés comercial (LITZ, 1997). Sin embargo, la descripción morfológica y agronómica del mango en México es escasa. CHÁVEZ et al. (2001) describieron algunas características agronómicas de interés comercial en variedades de mango introducidas y cultivadas en Michoacán, México. Dichos autores observaron que las variedades Haden, Tommy Atkins, Manila e Irwin son resistentes a la mosca de la fruta (Anastrepha ludens, Ceratitis capitata), a la roña (Elsinoe mangiferae) y la antracnosis (Colletotrichum gloeosporioides Penz). No se conocen más estudios de tipo morfológico y/o agronómico de mango nativo o mejorado a pesar de que el germoplasma se ha diversificado desde su introducción a México. El objetivo de este trabajo fue ubicar y caracterizar morfológicamente mangos nativos de la región Soconusco de Chiapas, México.

\section{MATERIAL Y MÉTODOS}

Se identificaron 36 registros de mango nativos de la región Soconusco, ubicada al sureste del estado de Chiapas, México (Tabla 1, Figura 1). De enero a mayo de 2006, se midieron 28 características morfológicas de 15 frutos y semillas de cada accesión de acuerdo con la lista de descriptores del IPGRI (1998) (Tabla 2). La edad mínima de los árboles incluidos en el estudio fue de 30 años (GÁLVEZ-LÓPEZ et al., 2006). Se calcularon, con los datos de cada registro, la media, rango, varianza, desviación estándar y coeficiente de variación de cada característica morfológica medida con el programa de cómputo Statistica versión 5.1 para Windows (STATSOFT, 1997).

\section{RESULTADOS}

Se observó una amplia diversidad morfológica en los frutos y semillas del germoplasma de mango de Chiapas (Figura 1). En este trabajo se encontraron características cualitativas no reportadas por en los descriptores morfológicos del IPGRI (1998), tales como los colores de la cáscara del fruto maduro na- 
Tabla 1. Coordenadas del área de ubicación de 36 mangos criollos de Chiapas, México.

\begin{tabular}{|c|c|c|c|c|c|}
\hline \multirow[b]{2}{*}{ Registro } & \multirow[b]{2}{*}{ Nombre común } & \multirow[b]{2}{*}{ Origen } & \multicolumn{3}{|c|}{ Localización geográfica } \\
\hline & & & Latitud Norte & Longitud Oeste & Altitud (msnm) \\
\hline TCH01 & Agua & Tuxtla Chico & $14^{\circ} 53^{\prime} 43^{\prime \prime}$ & $92^{\circ} 13^{\prime} 28^{\prime \prime}$ & 189 \\
\hline TCH02 & Sin Nombre 1 & “ & $14^{\circ} 53^{\prime} 41^{\prime \prime}$ & $92^{\circ} 13^{\prime} 30^{\prime \prime}$ & 203 \\
\hline TCH03 & Sin Nombre 2 & “ & $14^{\circ} 53^{\prime} 43^{\prime \prime}$ & $92^{\circ} 13^{\prime} 29^{\prime \prime}$ & 180 \\
\hline TCH04 & Oro & “ & $14^{\circ} 53^{\prime} 24^{\prime \prime}$ & $92^{\circ} 12^{\prime} 26^{\prime \prime}$ & 173 \\
\hline TCH05 & Coche & “ & $14^{\circ} 53^{\prime} 25^{\prime \prime}$ & $92^{\circ} 12^{\prime} 27^{\prime \prime}$ & 181 \\
\hline TCH06 & Amatillo & “ & $14^{\circ} 53^{\prime} 25^{\prime \prime}$ & $92^{\circ} 12^{\prime} 16^{\prime \prime}$ & 182 \\
\hline TCH07 & Alcanforado & “ & $14^{\circ} 53^{\prime} 25^{\prime \prime}$ & $92^{\circ} 12^{\prime} 15^{\prime \prime}$ & 188 \\
\hline TCH08 & Platano & “ & $14^{\circ} 53^{\prime} 24^{\prime \prime}$ & $92^{\circ} 12^{\prime} 15^{\prime \prime}$ & 171 \\
\hline TCH09 & Sin Nombre 3 & “ & 14º53’06” & $92^{\circ} 12^{\prime} 15^{\prime \prime}$ & 176 \\
\hline TCH10 & Pińa & “ & $14^{\circ} 53^{\prime} 06^{\prime \prime}$ & $62^{\circ} 12^{\prime} 26^{\prime \prime}$ & 164 \\
\hline TCH11 & Manzana & “ & 14º53’06” & $92^{\circ} 12^{\prime} 26^{\prime \prime}$ & 164 \\
\hline TCH12 & Piña & “ & 14º53’06” & $92^{\circ} 12^{\prime} 26^{\prime \prime}$ & 164 \\
\hline TCH13 & Jocote o Cachetio & “ & $14^{\circ} 53^{\prime} 09^{\prime \prime}$ & $92^{\circ} 12^{\prime} 30^{\prime \prime}$ & 177 \\
\hline TCH14 & Ajo & “ & $14^{\circ} 53^{\prime} 49^{\prime \prime}$ & $92^{\circ} 12^{\prime} 48^{\prime \prime}$ & 187 \\
\hline ESC01 & Piña & Escuintla & $15^{\circ} 19^{\prime} 06^{\prime \prime}$ & $92^{\circ} 39^{\prime} 32^{\prime \prime}$ & 75 \\
\hline ESC02 & Amatillo & “ & $15^{\circ} 19^{\prime} 06^{\prime \prime}$ & $92^{\circ} 39^{\prime} 03^{\prime \prime}$ & 75 \\
\hline ESC03 & Coche & “ & $15^{\circ} 19^{\prime} 05^{\prime \prime}$ & $92^{\circ} 39^{\prime} 32^{\prime \prime}$ & 75 \\
\hline ESC04 & Pomarrosa & “ & $15^{\circ} 19^{\prime} 05^{\prime \prime}$ & $92^{\circ} 39^{\prime} 32^{\prime \prime}$ & 82 \\
\hline ESC05 & Canela & “ & $15^{\circ} 19^{\prime} 03^{\prime \prime}$ & $92^{\circ} 39^{\prime} 29^{\prime \prime}$ & 85 \\
\hline ESC06 & Tapanero & “ & $15^{\circ} 19^{\prime} 03^{\prime \prime}$ & $92^{\circ} 39^{\prime} 29^{\prime \prime}$ & 85 \\
\hline ESC07 & Manzana Chico & “ & $15^{\circ} 19^{\prime} 03^{\prime \prime}$ & $92^{\circ} 39^{\prime} 29^{\prime \prime}$ & 85 \\
\hline ESC08 & Manilon & “ & $15^{\circ} 19^{\prime} 03^{\prime \prime}$ & $92^{\circ} 39^{\prime} 26^{\prime \prime}$ & 74 \\
\hline ESC09 & Manzana Grande & “ & $15^{\circ} 19^{\prime} 00^{\prime \prime}$ & $92^{\circ} 39^{\prime} 26^{\prime \prime}$ & 86 \\
\hline ESC10 & Viejita & “ & $15^{\circ} 18^{\prime} 60^{\prime \prime}$ & $92^{\circ} 39^{\prime} 24^{\prime \prime}$ & 88 \\
\hline ESC11 & Oro & “ & $15^{\circ} 19^{\prime} 01^{\prime \prime}$ & $92^{\circ} 39^{\prime} 24^{\prime \prime}$ & 81 \\
\hline ESC12 & Tecolote & “ & $15^{\circ} 19^{\prime} 01^{\prime \prime}$ & $92^{\circ} 39^{\prime} 22^{\prime \prime}$ & 80 \\
\hline ESC13 & Pepino & “ & $15^{\circ} 18^{\prime} 53^{\prime \prime}$ & $92^{\circ} 39^{\prime} 20^{\prime \prime}$ & 72 \\
\hline ESC14 & Pija & “ & $15^{\circ} 19^{\prime} 00^{\prime \prime}$ & $92^{\circ} 39^{\prime} 15^{\prime \prime}$ & 93 \\
\hline ESC15 & Melón & “ & $15^{\circ} 19^{\prime} 00^{\prime \prime}$ & $92^{\circ} 39^{\prime} 15^{\prime \prime}$ & 96 \\
\hline HUE01 & Tecolote & Huehuetán & $15^{\circ} 01^{\prime} 53^{\prime \prime}$ & $92^{\circ} 21^{\prime} 09^{\prime \prime}$ & 332 \\
\hline HUE02 & Manililla & “ & 15०01'39" & $92^{\circ} 20^{\prime} 31^{\prime \prime}$ & 150 \\
\hline HUE03 & Alcanfor & “ & $15^{\circ} 01^{\prime} 42^{\prime \prime}$ & $92^{\circ} 20^{\prime} 42^{\prime \prime}$ & 340 \\
\hline HUE04 & Amate & “ & $15^{\circ} 01^{\prime} 46^{\prime \prime}$ & $92^{\circ} 20^{\prime} 53^{\prime \prime}$ & 335 \\
\hline PIJ01 & Papaya & Pijijiapan & $17^{\circ} 01^{\prime} 46^{\prime \prime}$ & $92^{\circ} 41^{\prime} 57^{\prime \prime}$ & 62 \\
\hline PIJ02 & Piña & “ & $17^{\circ} 01^{\prime} 46^{\prime \prime}$ & $92 \circ 41^{\prime} 57^{\prime \prime}$ & 62 \\
\hline PIJ03 & Cuero & “ & $17^{\circ} 01^{\prime} 46^{\prime \prime}$ & $92^{\circ} 41^{\prime} 57^{\prime \prime}$ & 62 \\
\hline PIJ04 & Agua & “ & $17^{\circ} 01^{\prime} 15^{\prime \prime}$ & $92 \circ 41^{\prime} 57^{\prime \prime}$ & 62 \\
\hline TAP01 & Ataulfo $1(70)$ & Tapachula & $14^{\circ} 54^{\prime} 15^{\prime \prime}$ & $92^{\circ} 15^{\prime} 34^{\prime \prime}$ & 75 \\
\hline TAP02 & Ataulfo 2 (70) & “ & 140 54'14" & $92^{\circ} 15^{\prime} 34^{\prime \prime}$ & 75 \\
\hline TAP03 & Ataulfo 3 (50) & “ & $14^{\circ} 54^{\prime} 14^{\prime \prime}$ & $92^{\circ} 15^{\prime} 34^{\prime \prime}$ & 75 \\
\hline TAP04 & Ataulfo 4 (30) & “ & $14054 ’ 14^{\prime \prime}$ & $92^{\circ} 15^{\prime} 34^{\prime \prime}$ & 75 \\
\hline
\end{tabular}




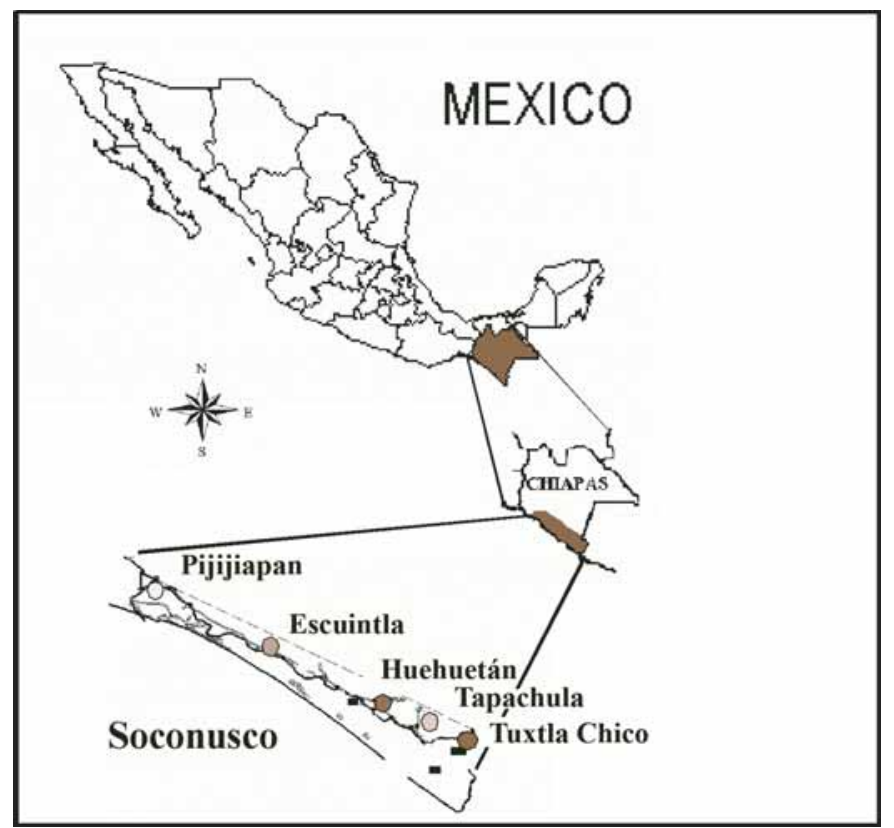

Figura 1. Mapa que indica la ubicación aproximada de las cinco localidades donde se colectó el germoplasma de mango nativo de la región Soconusco en Chiapas, México.

\begin{tabular}{|c|c|c|c|c|c|}
\hline Nombre & Fruto maduro & Nombre & Fruto maduro & Nombre & Fruto maduro \\
\hline Manililla & & Plátano & & Agua & \\
\hline Canela & & Viejita & & Plátano & \\
\hline Coche & & Tecolote & & Sin nombre & \\
\hline Amatillo & & Pepino & & Ajo & \\
\hline Cachetio & & Manzana & & Sin Nombre & \\
\hline Oro & & Alcanfor & & Ataulfo 50 & \\
\hline
\end{tabular}

Figura 2. Aspecto de los frutos maduros de algunos registros de mango nativas del Soconusco, Chiapas, México. 
Tabla 2. Características morfológicas medidas en germoplasma criollo de mango de Chiapas.

\begin{tabular}{|c|c|c|}
\hline & Característica & Escala demedición \\
\hline \multirow[t]{25}{*}{ Fruto } & Longitud & Centímetro \\
\hline & Ancho & Centimetro \\
\hline & Grosor & Centímetro \\
\hline & Forma & Ordinal: oblonga $=1$, eliptica $=2$, redonda $=3$ \\
\hline & Peso & Gramo \\
\hline & $\begin{array}{l}\text { Color de la cáscara del fruto } \\
\text { maduro }\end{array}$ & $\begin{array}{l}\text { Ordinal: rojo }=1 \text {, amarillo }=2 \text {, verde-amarillento }=3 \text {, verde }=4 \text {, } \\
\text { otra }=5 \text {, naranja }=6 \quad \text { a , rojo amarillento }=7 \quad \text { a , amarillo con puntos }\end{array}$ \\
\hline & & rojos $=8^{a}$, amarillo con puntos verdes $=9$ \\
\hline & Grosor de la cáscara & $\begin{array}{l}\text { Ordinal: delgada }=1, \text { delgada media }=2, \text { gruesa }=3, \text { muy } \\
\text { grues } a=4\end{array}$ \\
\hline & Peso de la cáscara & Gramo \\
\hline & Textura de la cáscara & Ordinal: lisa $=1$, rugosa $=2$ \\
\hline & $\begin{array}{l}\text { Contenido de la pulpa (radio de } \\
\text { la pulpa hasta el hueso) }\end{array}$ & Gramos \\
\hline & Textura de la pulpa & Ordinal: firme $=3$, suave $=5$, jugosa $=7$ \\
\hline & Adherencia de la piel a la pulpa & Ordinal: ausente $($ libre $)=0$, presente $($ adherida $)=(+)$ \\
\hline & Fibra en la pulpa & Ordinal: ausente $=0$, presente $(+)$ \\
\hline & Cantidad de fibra & Ordinal: escasa $=3$, abundante $=7$ \\
\hline & Longitud de fibras & Ordinal: corta $=3$, media $=5$, larga $=7$ \\
\hline & Inserción del tallo & Ordinal: vertical $=1$, Oblicua $=2$ \\
\hline & Tipo de punta & $\begin{aligned} & \text { Ordinal: ausente }= 0 \text {, un punto } \\
&=1 \text {, prominente }=2 \text {, en forma de } \\
& \text { pezón }=3\end{aligned}$ \\
\hline & Seno & Ordinal: ausente $=0$, presente $=(+)$ \\
\hline & Tipo de seno & Ordinal: ausente $=0$, ligeramente profundo $=1$, profundo $=2$ \\
\hline & Surco & Ordinal: ausente $=0$, presente $=(+)$ \\
\hline & Hombros & $\begin{aligned} \text { Ordinal: al nivel }= & 1 \text {, dorsal más alto que el ventral }=2 \text {, ventral } \\
& \text { más alto que el dorsal }=3 \text { a }\end{aligned}$ \\
\hline & Tipo de hombros & $\begin{array}{l}\text { Ordinal: declive abrupto }=1 \text {, terminación en curva larga }=2, \\
\text { pronunciado y redondeado }=3 \quad \text { a }\end{array}$ \\
\hline & Ápice & Ordinal: agudo $=1$, obtuso o redondo $=2$ \\
\hline & Cavidad basal & Ordinal: ausente $=0$, presente $=(+)$ \\
\hline \multirow[t]{6}{*}{ Semilla } & Longitud & Centímetro \\
\hline & Peso & Gramo \\
\hline & Venas & Ordinal: a nivel de superficie $=1$, hundido $=2$, elevado $=3$ \\
\hline & Patrones de venación & $\begin{aligned} \text { Ordinal: } \text { paralelo }=1, \text { bifurcado } & =2 \text {, ambos paralelo y } \\
\text { bifurcado } & =3{ }^{\text {a }}\end{aligned}$ \\
\hline & Fibra & Ordinal: ausente $=0$, presente $=(+)$, escasa $=1$ \\
\hline & Longitud de las fibras & Milímetro \\
\hline
\end{tabular}

a Características no consideradas en IPGRI (1998). 
Tabla 3. Resumen de las características cualitativas de 36 registros de mango criollo de Chiapas.

\begin{tabular}{|c|c|c|c|c|c|c|c|c|}
\hline Accesión & Forma & $\begin{array}{c}\text { Color de la } \\
\text { cáscara del fruto } \\
\text { maduro }\end{array}$ & $\begin{array}{c}\text { Textura de la } \\
\text { pulpa }\end{array}$ & Tipo de punta & Hombros & $\begin{array}{l}\text { Tipo de } \\
\text { hombros }\end{array}$ & $\begin{array}{l}\text { Patrones de } \\
\text { venación de } \\
\text { semilla }\end{array}$ & Fibra en semilla \\
\hline TCH01 & 1 & 2 & 7 & 1 & 3 & 3 & 1 & + \\
\hline $\mathrm{TCH} 02$ & 3 & 3 & 7 & 1 & 1 & 3 & 2 & + \\
\hline $\mathrm{TCH} 03$ & 3 & 6 & 3 & 0 & 3 & 1 & 1 & 0 \\
\hline TCHO4 & 2 & 2 & 7 & 2 & 2 & 2 & 1 & + \\
\hline TCH05 & 3 & 2 & 5 & 1 & 1 & 3 & 1 & 0 \\
\hline TCH06 & 3 & 2 & 5 & 0 & 1 & 2 & 3 & + \\
\hline TCH07 & 1 & 2 & 5 & 1 & 1 & 2 & 2 & + \\
\hline TCH08 & 1 & 4 & 3 & 3 & 3 & 1 & 1 & + \\
\hline TCH10 & 3 & 2 & 7 & 0 & 1 & 2 & 1 & + \\
\hline TCH11 & 3 & 7 & 3 & 0 & 2 & 3 & 1 & + \\
\hline $\mathrm{TCH} 12$ & 3 & 2 & 7 & 0 & 1 & 2 & 1 & + \\
\hline TCH13 & 2 & 1 & 3 & 1 & 1 & 1 & 1 & 1 \\
\hline TCH14 & 1 & 3 & 3 & 1 & 1 & 3 & 3 & 1 \\
\hline $\mathrm{ESC} 01$ & 3 & 2 & 7 & 0 & 1 & 2 & 1 & + \\
\hline $\mathrm{ESC} 02$ & 3 & 2 & 3 & 0 & 1 & 3 & 1 & + \\
\hline $\mathrm{ESC} 03$ & 2 & 3 & 3 & 1 & 2 & 3 & 1 & + \\
\hline $\mathrm{ESC} 04$ & 1 & 3 & 3 & 0 & 2 & 3 & 1 & + \\
\hline $\mathrm{ESC} 05$ & 2 & 4 & 7 & 1 & 1 & 1 & 1 & + \\
\hline $\mathrm{ESC} 07$ & 3 & 7 & 3 & 0 & 2 & 3 & 1 & + \\
\hline $\mathrm{ESC} 09$ & 3 & 7 & 3 & 0 & 2 & 3 & 1 & + \\
\hline $\mathrm{ESC} 10$ & 2 & 8 & 7 & 0 & 2 & 2 & 2 & + \\
\hline ESC11 & 2 & 2 & 7 & 2 & 2 & 2 & 1 & + \\
\hline $\mathrm{ESC} 12$ & 3 & 9 & 5 & 0 & 3 & 1 & 2 & + \\
\hline $\mathrm{ESC} 13$ & 1 & 7 & 5 & 0 & 1 & 1 & 1 & + \\
\hline ESC14 & 1 & 4 & 7 & 3 & 3 & 1 & 1 & + \\
\hline HUE01 & 3 & 3 & 5 & 1 & 3 & 3 & 2 & + \\
\hline HUE02 & 1 & 2 & 5 & 2 & 3 & 2 & 1 & + \\
\hline HUE03 & 1 & 2 & 5 & 1 & 1 & 2 & 2 & + \\
\hline HUE04 & 3 & 2 & 3 & 0 & 1 & 3 & 1 & + \\
\hline PIJ01 & 1 & 2 & 7 & 3 & 1 & 2 & 1 & + \\
\hline PIJ02 & 3 & 2 & 7 & 0 & 1 & 2 & 1 & + \\
\hline PIJ04 & 1 & 2 & 7 & 1 & 3 & 3 & 1 & + \\
\hline TAP01 & 1 & 2 & 3 & 2 & 3 & 3 & 1 & + \\
\hline $\mathrm{TAP} 02$ & 1 & 2 & 3 & 2 & 3 & 2 & 1 & + \\
\hline TAP03 & 1 & 2 & 5 & 2 & 3 & 2 & 1 & + \\
\hline TAP04 & 1 & 2 & 5 & 2 & 3 & 2 & 1 & + \\
\hline
\end{tabular}

Las claves son de acuerdo con IPGRI (1998) (Tabla 2). 
Tabla 4. Resumen de las características cuantitativas de 36 registros de mango criollo de Chiapas.

\begin{tabular}{|c|c|c|c|c|c|c|c|}
\hline Accesión & Longitud (cm) & Ancho (cm) & Grosor $(\mathrm{cm})$ & Peso (g) & $\begin{array}{l}\text { Contenido de la } \\
\text { pulpa (radio de } \\
\text { la pulpa hasta el } \\
\text { hueso) (g) }\end{array}$ & $\begin{array}{l}\text { Longitud de } \\
\text { semilla }(\mathrm{cm})\end{array}$ & $\begin{array}{c}\text { peso de semilla } \\
(\mathrm{g})\end{array}$ \\
\hline TCH01 & 12.6 & 8.7 & 7.2 & 428 & 2.9 & 9.2 & 30.7 \\
\hline TCH02 & 9.6 & 7.4 & 6.3 & 246 & 2.5 & 9.7 & 26.0 \\
\hline $\mathrm{TCH} 03$ & 12.5 & 8.9 & 7.1 & 488 & 5.1 & 8.1 & 30.0 \\
\hline TCH04 & 10.1 & 6.3 & 5.1 & 183 & 0.9 & 7.9 & 23.4 \\
\hline TCH05 & 8.4 & 6.4 & 5.6 & 308 & 2.0 & 6.7 & 23.6 \\
\hline ТCH06 & 10.0 & 7.8 & 6.6 & 306 & 1.4 & 9.6 & 23.2 \\
\hline ТCH07 & 9.6 & 6.5 & 5.6 & 187 & 2.0 & 8.6 & 17.1 \\
\hline TCH08 & 14.0 & 6.9 & 5.7 & 293 & 2.9 & 10.9 & 18.0 \\
\hline TCH10 & 8.7 & 6.6 & 5.4 & 189 & 1.6 & 7.3 & 15.1 \\
\hline TCH11 & 7.8 & 7.2 & 6.7 & 221 & 2.1 & 6.7 & 20.3 \\
\hline TCH12 & 8.7 & 6.6 & 5.4 & 189 & 1.6 & 7.3 & 15.1 \\
\hline TCH13 & 8.2 & 5.7 & 5.5 & 130 & 1.9 & 6.6 & 16.8 \\
\hline TCH14 & 13.0 & 8.8 & 6.9 & 440 & 2.9 & 9.2 & 26.0 \\
\hline ESC01 & 8.7 & 6.6 & 5.4 & 189 & 1.6 & 7.3 & 15.1 \\
\hline $\mathrm{ESC} 02$ & 9.0 & 5.0 & 6.0 & 210 & 1.4 & 7.7 & 24.1 \\
\hline $\mathrm{ESC} 03$ & 8.3 & 6.1 & 3.9 & 143 & 1.3 & 7.1 & 20.9 \\
\hline ESC04 & 10.3 & 7.1 & 6.9 & 254 & 1.1 & 8.5 & 29.6 \\
\hline ESC05 & 8.7 & 5.3 & 4.8 & 127 & 1.2 & 7.2 & 14.6 \\
\hline ESC07 & 7.8 & 7.2 & 6.7 & 221 & 2.1 & 6.7 & 20.3 \\
\hline ESC09 & 7.8 & 7.2 & 6.7 & 221 & 2.1 & 6.7 & 20.3 \\
\hline $\mathrm{ESC} 10$ & 8.7 & 5.9 & 4.8 & 135 & 1.5 & 7.5 & 15.6 \\
\hline ESC11 & 10.1 & 6.4 & 5.1 & 183 & 0.9 & 7.9 & 23.4 \\
\hline $\mathrm{ESC} 12$ & 10.4 & 7.9 & 6.0 & 316 & 1.5 & 9.2 & 21.0 \\
\hline ESC13 & 10.2 & 5.4 & 4.8 & 141 & 1.7 & 8.7 & 11.1 \\
\hline ESC14 & 14.0 & 6.9 & 5.7 & 293 & 2.9 & 10.9 & 18.0 \\
\hline HUE01 & 10.6 & 7.9 & 6.5 & 293 & 2.2 & 9.44 & 16.6 \\
\hline HUE02 & 10.7 & 6.7 & 5.9 & 206 & 2.4 & 10.2 & 16.2 \\
\hline HUE03 & 9.6 & 6.5 & 5.6 & 187 & 2.1 & 8.6 & 17.1 \\
\hline HUE04 & 9.0 & 5.0 & 6.0 & 210 & 1.4 & 7.7 & 24.1 \\
\hline PIJ01 & 12.6 & 7.3 & 6.2 & 297 & 1.7 & 9.9 & 24.5 \\
\hline PIJ02 & 8.7 & 6.6 & 5.4 & 189 & 1.6 & 7.3 & 15.1 \\
\hline PIJ04 & 12.6 & 8.7 & 7.2 & 428 & 2.9 & 9.2 & 30.7 \\
\hline TAP01 & 11.5 & 7.9 & 7.1 & 374 & 3.3 & 8.9 & 18.1 \\
\hline TAP02 & 11.2 & 7.0 & 6.3 & 281 & 2.7 & 9.9 & 18.4 \\
\hline TAP03 & 11.1 & 7.1 & 6.3 & 275 & 2.5 & 8.6 & 11.8 \\
\hline TAP04 & 11.1 & 6.9 & 6.0 & 245 & 2.9 & 8.9 & 14.1 \\
\hline
\end{tabular}


Tabla 5. Resumen estadístico de las características cuantitativas de fruto de 36 registros de mango criollo de Chiapas.

\begin{tabular}{lccccc}
\hline & \multicolumn{5}{c}{ Característica } \\
\cline { 2 - 5 } Variable & Media & Rango & Varianza & $\begin{array}{c}\text { Desviación } \\
\text { estándar }\end{array}$ & $\begin{array}{c}\text { Coeficiente } \\
\text { de variación } \\
(\%)\end{array}$ \\
\hline Longitud del fruto & 10.2 & 6.3 & 3.2 & 1.8 & 17.5 \\
Ancho del fruto & 6.9 & 3.9 & 1.0 & 1.0 & 14.7 \\
Grosor del fruto & 6.0 & 3.3 & 0.6 & 0.8 & 13.3 \\
Peso del fruto & 251 & 362.2 & 8533.3 & 92.4 & 36.9 \\
Contenido de pulpa & 2.1 & 4.2 & 0.7 & 0.8 & 40.6 \\
Longitud de la semilla & 8.4 & 4.3 & 1.5 & 1.2 & 14.7 \\
Peso de la semilla & 20.2 & 19.6 & 28.2 & 5.3 & 26.3 \\
\hline
\end{tabular}

ranja, rojo amarillento, amarillo con puntos rojos y amarillo con puntos verdes; hombros con posición ventral más alta que la dorsal; tipo de hombro pronunciado y redondeado; semillas con patrones de venación paralelo y bifurcado y semilla con cantidad de fibra escasa (Tabla 2). De las 21 características cualitativas registradas sólo una mostró una clase fenotípica, mientras el resto mostró de dos a ocho clases y la característica más diversa fue el color de la cáscara del fruto. Se observaron agrupaciones por características en algunas poblaciones como los mangos 'Ataulfo' de Tapachula mismos que muestran forma de fruto oblonga, punta prominente y hombros más altos que el dorsal; los mangos de Huehuetán, Pijijiapan y Tapachula exhiben cáscara color verdeamarillento; fruto con textura firme, suave y jugosa de la pulpa; con frutos con terminación en curva larga y pronunciada y redondeada (Tabla 3). En general, los mangos mejorados "Ataulfo" de Tapachula mostraron los valores más altos en tamaño de fruto (largo, ancho, grosor de mesocarpio, peso de pulpa y peso total), así como en longitud y peso de semilla. No obstante, algunos registros de mango nativo de Chiapas mostraron valores similares e incluso superiores a dichos mangos mejorados. Altos pesos de fruto mostraron los registros 'Agua', 'Sin Nombre-2', 'Amate', 'Tecolote', 'Plátano' y 'Manzana'; mayores contenidos de pulpa se observaron en 'Sin nombre-2', 'Agua' y 'Plátano'. Los mangos nativos de Chiapas con características del fruto similares a 'Ataulfo' fueron 'Ajo' y 'Sin Nombre-2' de Tuxtla Chico (Tabla 4). Las características cuantitativas con mayores coeficientes de variación (>36 \%) fueron grosor de mesocarpio y peso del fruto; mientras que las menos variables (coeficiente de variación $<14$ $\%$ ) fueron grosor y ancho del fruto y longitud de la semilla (Tabla 5).

\section{DISCUSIÓN}

En este trabajo se encontraron características cualitativas de fruto diferentes a las reportadas en el germoplasma Asiático, del que proviene el germoplasma Mexicano e inclusive, no consignadas en los descriptores de mango del IPGRI (1998). La amplia variabilidad morfológica observada en el fruto del germoplasma de mango de Chiapas sugiere la constante recombinación genética entre el germoplasma introducido a México y a Chiapas en particular. En Asia, los mangos con cáscaras rojas y amarillas son los más apreciados en el mercado (HUMAN \& RHEEDER, 2004); mientras que en Europa y EUA se prefieren mangos con colores amarillos a rojos (RAMOS, 2000). En Chiapas se 
observan mangos nativos con dichos colores de cáscara (roja: cachetio, manzana, pepino; amarilla: agua, coche, amate, viejita, oro, manililla, papaya y ataulfo). También, se observaron cáscaras de colores verde-amarillento, rojo amarillento, amarillo con puntos rojos, amarillo con puntos verdes. La gama de colores de frutos observados podría tener potencial para su explotación comercial si se dirige hacia mercados específicos. Por ejemplo, los frutos color naranja observado en el mango 'sin nombre2' de Tuxtla Chico, podrían ser atractivos para los consumidores de México y el extranjero. Las características fenotípicas del fruto de $M$. indica definen su utilidad potencial para fines productivos y comerciales, debido a que estas suelen ser importantes (HUMAN \& RHEEDER, 2004) para definir su destino, ya sea industrial (fabricación de jugos y néctares) o para el consumo en fresco. Por lo tanto, y dadas las características nuevas observadas en el germoplasma de Chiapas, dicho material genético tiene amplio potencial comercial y para el mejoramiento genético de la especie en Chiapas y México en general. Respecto a las características cuantitativas, los mayores porcentajes de variabilidad se observaron en contenido de pulpa y peso del fruto, mismos que se asocian con productividad y que son propiedades deseables en mango destinados al consumo en fresco (RAMOS, 2003). CHÁVEZ et al. (2001) determinaron que los pesos promedio de fruto de variedades comerciales de mango mejorado cultivadas en Michoacán, México variaron de 200 a 800 g. En el germoplasma nativo de Chiapas exhibió pesos de fruto de 126 a 500g en promedio, destacando por los valores mayores los registros 'Agua', 'Sin Nombre-2', 'Amate', 'Tecolote', 'Plátano' y 'Manzana'. Los mangos con mayor contenido de pulpa en frutos fueron 'Sin nombre-2', 'Ataulfo2', 'Agua', 'Plátano' y 'Ataulfo-4'. Para el mercado en fresco se prefieren mangos con baja cantidad de fibra y fibra de menor longitud, así como mayor longitud, ancho, grosor, peso y contenido de pulpa en el fruto (RAMOS, 2000; GRUPO PM, 2000). En este estudio, los mangos que presentaron estas características deseables fueron los 'Ataulfo' de Tapachula y 'Ajo' y 'Sin Nombre-2' de Tuxtla Chico. El IPGRI (1989) indica que los mangos nativos de ciertas zonas presentan ventajas tales como alta pro- ductividad y tolerancia a condiciones desfavorables (suelos mal drenados, carencia de nutrimentos, resistencia a plagas y enfermedades) en comparación con las variedades mejoradas e introducidas. Los resultados de este trabajo indican que los mangos nativos de Chiapas son una fuente de germoplasma importante para México, de modo que se considera prioritaria la conservación y propagación de este acervo genético pues podría constituirse como fuente para la producción de nuevas variedades con características deseables para el cultivo con fines comerciales.

\section{AGRADECIMIENTOS}

Los autores agradecen al Instituto Politécnico Nacional (proyecto CGPI-20050084) y al Fondo Mixto-Gobierno del Estado de Tamaulipas el financiamiento de este trabajo, así como a la Dra. M. L. Adriano-Anaya (Universidad Autónoma de Chiapas) por el apoyo en la descripción de campo del germoplasma. N. Mayek-Pérez es becario del Sistema Nacional de Investigadores, de la COFAAIPN y del programa EDI-IPN.

\section{REFERENCIAS BIBLIOGRAFICAS}

CHÁVEZ, C. X., A. VEGA, L. M. TAPIA \& S. MIRANDA. 2001. Mango, su manejo y producción en el trópico seco de México. INIFAP. Morelia, México. Pp. 17-25.

GÁLVEZ-LÓPEZ, D., M. L. ADRIANO-ANAYA, C. VILLARREAL-TREVIÑO, N. MAYEK-PÉREZ \& M. SALVADOR-FIGUEROA. 2007. Caracterización isoenzimática de mango criollo de Chiapas. Revista Chapingo, Serie Horticultura (en prensa).

GRUPO PM. 2000. El mango en el Mercado norteamericano de frutas y verduras. Disponible en: http://ie3global.oregonstate.edu/ie3/openings/ mexic3.html. [Fecha de consulta: 12 de Mayo de 2005].

HUMAN, C. F. \& S. RHEEDER. 2004. Mango breeding: results and successes. En: Proceedings of the Seventh International Mango Symposium. Section Tropical and Subtropical Fruits Working Group on Mango. Acta Horticulturae 645: 331-335. 
INSTITUTO INTERNACIONAL DE RECURSOS FITOGENÉTICOS (IPGRI). 1989. Descriptors of mango. Rome, Italy. Pp. 1-22.

JARAMILLO, S. \& M. BAENA. 2000. Conservación ex situ de recursos fitogenéticos. Instituto Internacional de Recursos Fitogenéticos (IPGRI). Madrid. 92 pp.

LITZ, R. E. 1997. The mango: botany, production, and uses. CAB International, Homestead, U. S. A. $587 \mathrm{pp}$.

RAMOS N. J. A. 2000. Competitividad del mango fresco de exportación. FIRA-Banco de México. Mazatlán, México. 40 pp.
RAMOS, N. J. A. 2003. Perspectivas de la Red Mango para el 2003. FIRA-Banco de México. México. Pp. 2-12.

SAGAR-INIFAP-PRODUCE. 2000. Logros y aportaciones de la investigación al cultivo del mango "Ataulfo" en Chiapas. Tapachula, México. 35 pp.

STATSOFT Inc. 1997. STATISTICA for Windows (Computer program manual). Release 5.1. Tulsa, OK, USA. 